\title{
Comparison of intraocular pressure as measured by three different non-contact tonometers and goldmann applanation tonometer for non-glaucomatous subjects
}

Seung Pil Bang ${ }^{\dagger}$, Chong Eun Lee ${ }^{\dagger}$ and Yu Cheol Kim ${ }^{*}$

\begin{abstract}
Background: To compare the measurement of intraocular pressure (IOP) among the three different non-contact tonometers (NCT) and the Goldmann applanation tonometer (GAT) for non-glaucomatous subjects.

Methods: In 52 eyes of 52 non-glaucomatous subjects, IOP was measured sequentially with the Canon TX-20P, the Nidek NT-530P, the Topcon CT-1P, and the GAT at the same time. We evaluated the IOP-measurement agreement among the tonometers as well as the factors affecting the measurements.

Results: A significant positive correlation was shown between the IOP values obtained with GAT and each NCT. The Canon TX-20P showed statistically the most significant agreement with the GAT (ICC 0.906, 95\% CI 0.837-0. 946). In an analysis of the Bland-Altman plots, the Canon TX-20P also showed the largest mean bias (1.38 mmHg) but the narrowest limits of agreement (LOA) (95\% LoA; $\pm 3.43 \mathrm{mmHg})$. The Topcon CT-1P showed the smallest mean bias $(0.48 \mathrm{mmHg})$ but the widest LoA (95\% LoA; $\pm 4.16 \mathrm{mmHg})$. The Topcon CT-1P and Nidek NT-530P both showed a significantly positive correlation between increasing central corneal thickness (CCT) and increasing IOP.

Conclusion: There was a statistically significant correlation between each of the three different NCT and the GAT measurements. IOP measured with the Canon TX-20P and Topcon CT-1P tended to be higher, and with the Nidek NT-530P lower, than with the GAT. Practitioners need to know the properties of their own NCTs and their respective measurement tendencies.
\end{abstract}

Keywords: Central corneal thickness, Goldmann applanation tonometer, Intraocular pressure, Non-contact tonometer

\section{Background}

Currently, the Goldmann applanation tonometer (GAT) is considered to be the gold standard for IOP measurement in clinical practice [1-6]. However, since their introduction, non-contact tonometers (NCTs) have become well established in clinical practice. NCT is a rapid, simple and objective method of intraocular pressure (IOP) measurement that can be performed by ancillary staff without the use of corneal anaesthesia [7].

\footnotetext{
* Correspondence: eyedr@dsmc.or.kr

${ }^{\dagger}$ Equal contributors

Department of Ophthalmology, Keimyung University School of Medicine,

Dongsan Medical Center, \#56, Dalseong-ro, Jung-gu, Daegu 41931, South Korea
}

Not all NCTs perform similarly. Although earlier models showed, relative to the GAT, a wider bias and a poor sensitivity for detection of elevated IOP $[1,3,7,8]$, the accuracies of subsequent models are much improved [9-18]. The Canon TX-20P, Nidek NT-530P, and Topcon CT-1P are the new automatic tonometers currently marketed. Perhaps surprisingly, there are in the literature, to our knowledge, few reports available on the performance of the Canon TX-20P or Nidek NT-530P [10-15], and none on the Topcon CT-1P. Also, various studies have compared just one or two of the NCTs with the GAT, and there have been few studies comparing the accuracy of various tonometers for non-glaucomatous healthy adult subjects $[10,14$, 19-21]. 
The purposes of this study were to evaluate the reliability of the three new NCTs and to compare their IOP measurements with those obtained by the GAT for nonglaucomatous subjects. For each tonometer, the relationships between the measured ocular parameters and the IOP measurements also were examined.

\section{Methods}

Fifty-two eyes of 52 non-glaucomatous healthy subjects aged 21 to 85 years were included in the study. The study was approved by the Institutional Review Board (IRB) of Keimyung University Dongsan Medical Center (IRB no. 2015-12-052) and was performed in accordance with the tenets of the Declaration of Helsinki. None of the subjects had any history of ocular pathology affecting IOP, refractive surgery or trauma, and none had worn contact lenses within the two-week period prior to IOP measurement. Subjects were excluded if they had regular astigmatism greater than 3.50 diopters (D) or any irregular astigmatism. To avoid the doubleorgan bias, one eye per individual was randomly included in the analysis using a table of random numbers for randomization [22]. In a fixed sequence, all of the subjects were examined with the three NCTs, the GAT and ultrasound pachymeter (850, Humphrey Instruments, Inc., San Leandro, CA, USA) to obtain IOP and central corneal thickness (CCT) measurements, respectively. According to the GAT IOP values, the eyes were categorized into low-teen $(<14 \mathrm{mmHg})$, mid-teen $(\geq 14$ and $\leq 17 \mathrm{mmHg}$ ) and high-teen $(>17 \mathrm{mmHg}$ ) groups.

IOP measurements were made by the same experienced ancillary staff using the three NCTs. The order in which the instruments were used was the Canon TX20P, followed by the Nidek NT-530P, the Topcon CT-1P and the GAT. Each of the tonometers was calibrated according to the manufacturer's guidelines prior to its use in this study. In manual measurements using the Nidek NT-530P, on the other hand, the operator aligns the cornea by superimposing a reflection of the target from the subject's cornea on a stationary ring and depresses the trigger when the cornea is aligned. In the present study, the mean of three measurements was used so as to avoid the effect of fluctuations caused by the cardiac pulse cycle.

IOP measurements were taken with the GAT (AT900; Haag-Streit, Köniz, Switzerland) according to the standard procedures. Before acquisition, one drop of $0.5 \%$ proparacaine hydrochloride eye drops (Alcaine ${ }^{\circledR}$, Alcon Laboratories Inc., Fort Worth, TX, USA) was instilled and a fluorescein strip was applied to the inferior conjunctival fornix. To avoid error introduced by topical anesthesia, the GAT was applied five minutes after eyedrop instillation [23]. The last IOP measurement was obtained using GAT to avoid a corneal-compression- induced aqueous outflow increase that would have affected subsequent IOP readings [24, 25]. Also, the mean of three measurements of GAT was used and each IOP readings were masked to the one clinician (SPB) performing the measurements. Between each instrumentation application, the subjects were allowed a five-minute rest period to recover from the aqueous outflow. All of the measurements were taken between 11:30 am and 1:30 pm in order to minimize the effects of diurnal IOP variation [26].

Pearson correlation analysis, the intraclass correlation coefficient (ICC), the paired t-test were used to assess the correlation, consistency and agreement among the IOP measurements provided by each instrument. We also constructed Bland-Altman plots using Medcalc version 15.2 (Ostend, Belgium) to compare the bias in the IOP measurements of each NCT relative to the GAT. Simple linear regression analysis was used to assess the correlations between CCT and the IOP measurements of each tonometer. The paired t-test was used to analyze the difference between the IOP measurements of each NCT and the GAT in each subgroup divided by the GAT IOP values. Data were analyzed using SPSS version 22 (IBM, Armonk, NY, USA).

\section{Results}

Of the 52 participants, 22 were male and 30 were female, with a mean age of $50.56 \pm 17.25$ years (range: $21-$ 85 years). The mean IOP across all subjects was $17.23 \pm 2.94 \mathrm{mmHg}$ (range: $11-21 \mathrm{mmHg}$ ) with the Canon TX-20P, $14.87 \pm 3.25 \mathrm{mmHg}$ (range: $8-21 \mathrm{mmHg}$ ) with the Nidek NT-530P, $16.33 \pm 3.01 \mathrm{mmHg}$ (range: 9-21 mmHg) with the Topcon CT-1P, and $15.85 \pm 3.05 \mathrm{mmHg}$ (range: $8-21 \mathrm{mmHg}$ ) with the GAT. The mean CCT as measured with the ultrasound pachymeter was 541.44 $\pm 28.49 \mu \mathrm{m}$ (range: 469-605 $\mu \mathrm{m})$.

\section{IOP comparisons}

A significant positive correlation was shown between the IOP values obtained with the GAT (G-IOP) and the Canon TX-20P (C-IOP: $R=0.829, p<0.001$ ), the Nidek NT-530P (N-IOP: $R=0.799, p<0.001$ ) and the Topcon CT-1P (T-IOP: $R=0.755, p<0.001$ ), respectively (Fig. 1 ). Also, a significantly high consistency by ICC was observed between the G-IOP and the C-IOP (ICC $=0.906,95 \% \mathrm{CI}$ : $0.837-0.946, p<0.001)$, the N-IOP (ICC $=0.887,95 \% \mathrm{CI}$ : $0.803-0.935, \mathrm{p}<0.001)$ and the T-IOP $(\mathrm{ICC}=0.861,95 \%$ CI: 0.757-0.920, p < 0.001), respectively.

Figure 2 shows a Bland-Altman scatterplot comparing the three NCT and GAT readings. The mean of the differences between the C-IOP and G-IOP was $1.38 \pm 1.75 \mathrm{mmHg}(95 \% \mathrm{LoA},-2.05$ to $+4.82 \mathrm{mmHg})$, between the N-IOP and G-IOP, $-0.98 \pm 2.00 \mathrm{mmHg}$ 

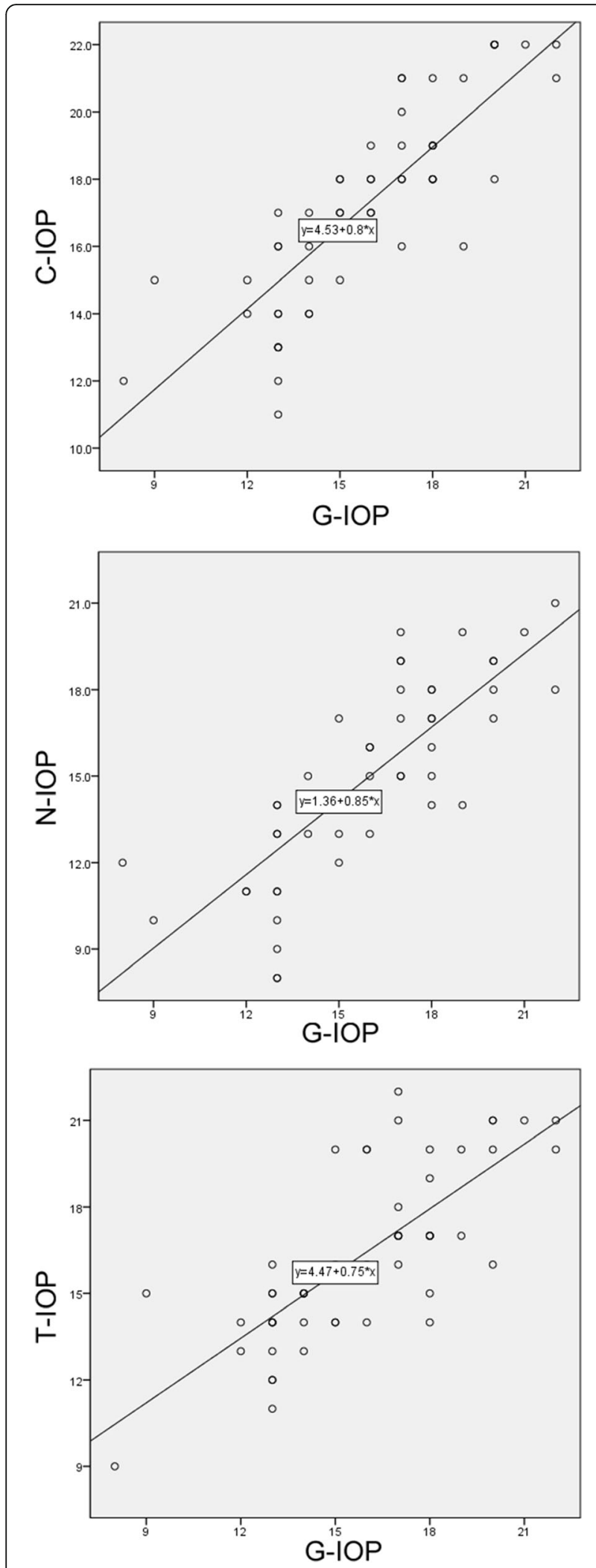

Fig. 1 Correlation analysis of intraocular pressure (IOP) measurements $(\mathrm{mmHg})$ taken with three different non-contact tonometers (NCTs) and Goldmann applanation tonometer (GAT). Significant positive correlations by Pearson correlation analysis were noted between the $I O P$ values measured by each of the NCTs and the GAT. C-IOP = IOP value measured by Canon NCT; N-IOP = IOP value measured by Nidek $\mathrm{NCT} ; \mathrm{T}-\mathrm{IOP}=\mathrm{IOP}$ value measured by Topcon NCT; G-IOP = IOP value measured by GAT

(95\% LoA, -4.91 to $+2.95 \mathrm{mmHg}$ ), and between the $\mathrm{T}$ IOP and G-IOP, $0.48 \pm 2.12 \mathrm{mmHg}(95 \% \mathrm{LoA},-3.67$ to $+4.63 \mathrm{mmHg}$ ). The narrowest $95 \% \mathrm{LoA}$, as indicative of the highest consistency, was that between the C-IOP and G-IOP. There was no significant linear relationship between the difference and the mean of each NCT/GAT pair, which fact indicated good equality between the 3 NCTs and the GAT.

There was a significant mean difference between, respectively, the Canon TX-20P and Nidek NT-530P and GAT IOP measurements but not between the Topcon CT-1P and GAT measurements. The mean difference between the C-IOP and G-IOP was $1.38 \mathrm{mmHg}$ $(p<0.001)$, and between the N-IOP and G-IOP, $0.98 \mathrm{mmHg}(p=0.001)$, as noted above (Table 1$)$. There was no significant mean difference between the IOP measurements of each NCT and those of the GAT in six of the nine subgroups divided by G-IOP; significant differences were found in the low- and mid-teen groups of the C-IOP and in the high-teen group of N-IOP. The mean differences between the C-IOP and G-IOP were $1.71 \mathrm{mmHg}(p=0.013)$ in the low-teen group and $1.77 \mathrm{mmHg}(p<0.001)$ in the mid-teen group. The mean difference between the N-IOP and G-IOP was $1.75 \mathrm{mmHg}(p=0.001)$ in the high-teen group (Table 2).

\section{Influence of CCT}

There was a significant positive correlation between CCT and T-IOP $(R=0.390, p=0.004)$, the IOP increasing with increasing corneal thickness; linear regression analysis showed a mean change of $0.4 \mathrm{mmHg}$ in $\mathrm{T}$-IOP per $10 \mu \mathrm{m}$ variation in CCT. Meanwhile, there was a weak positive correlation between CCT and N-IOP $(R=0.325, p=0.019)$; linear regression analysis also showed a mean change of $0.4 \mathrm{mmHg}$ in $\mathrm{N}$-IOP per $10 \mu \mathrm{m}$ variation in CCT. Contrastingly, no correlation was detected between CCT and C-IOP $(R=0.237$, $p=0.091)$ or G-IOP $(R=0.149, p=0.293)$, indicating no significant effect of CCT on C-IOP or G-IOP.

\section{Discussion}

This study compared the IOP-measurement performances of the three new NCTs with the GAT. With the same subjects at the same moment, the IOPs from each NCT were obtained and demonstrated the following 


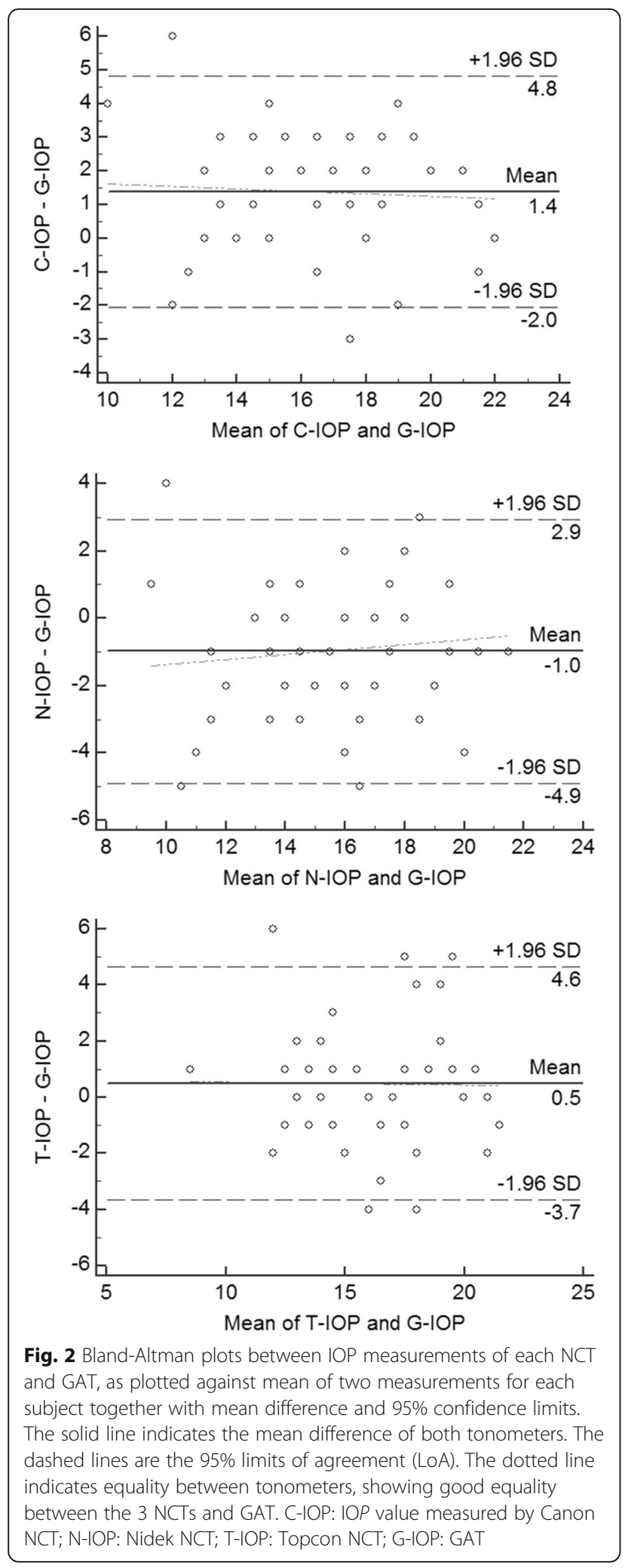

tendencies in terms of range: the IOP measurements with the Canon TX-20P and the Topcon CT-1P tended to be higher, whereas those with the Nidek NT-530P
Table 1 Pairwise comparative analyses between each NCT and GAT

\begin{tabular}{lllll}
\hline $\begin{array}{l}\text { NCT } \\
\text { device }\end{array}$ & $\begin{array}{l}\text { Mean }( \pm \mathrm{SD}) \\
(\mathrm{mmHg})\end{array}$ & $\begin{array}{l}\text { Mean difference }( \pm \mathrm{SD}) \\
\text { with G-IOP }\end{array}$ & 95\% LoA & $p$ value \\
\hline $\mathrm{C}-\mathrm{IOP}$ & $17.23 \pm 2.94$ & $1.38 \pm 1.75$ & -2.05 to 4.82 & $<0.001$ \\
$\mathrm{~N}-\mathrm{IOP}$ & $14.87 \pm 3.25$ & $-0.98 \pm 2.00$ & -4.91 to 2.95 & $\mathbf{0 . 0 0 1}$ \\
T-IOP & $16.33 \pm 3.01$ & $0.48 \pm 2.12$ & -3.67 to 4.63 & 0.108 \\
\hline
\end{tabular}

C-IOP IOP value measured by Canon NCT, N-IOP IOP value measured by Nidek NCT, T-IOP IOP value measured by Topcon NCT, G-IOP IOP value measured by GAT, LoA limits of agreement

$P$ value by paired t-test

tended to be lower than with the GAT. Considering the order of the tonometer measurements, the greatest IOP values of C-IOP could be due to the 'massage effect', though the NCTs were less affected than was the GAT $[24,25]$, and regardless, the five-minute interval between each measurement, enough time to recover from enhanced aqueous outflow due to corneal compression, would minimize such effect.

In terms of study samples, the mean IOPs recorded using the four tonometers were close to those obtained by other authors for healthy subjects $(15-16 \mathrm{mmHg}$ on average, SD 2.5-3.0 $\mathrm{mmHg}$ ) [3, 27]. Similarly, the mean CCT of $541.4 \mu \mathrm{m}$ determined using the ultrasound pachymeter was close to the population mean of $530.9 \mu \mathrm{m}$ and within the range found in normal Korean subjects [28].

The relationships observed between the CCT and IOP measurements obtained by the Nidek NT-530P and Topcon CT-1P tonometers are in agreement with the general assumption that CCT affects NCT readings, showing a positive correlation. According to previous reports, CCT affects both GAT and NCT readings [29, 30], the latter being more affected than the former [30-32]. The moderate correlations observed in this study ( $R=0.325$ and 0.390$)$ could be attributed to the limited range of CCT in our population $(469-605 \mu \mathrm{m})$. Other authors argue that for corneas with a CCT less than $575 \mu \mathrm{m}$, IOP measurements are unaffected [5, 33]. In our study population, only 6 of the 52 eyes showed a CCT greater than $575 \mu \mathrm{m}$.

Through Pearson correlation analysis (Fig. 1), we observed a highly significant relationship between the pressure readings provided by each NCT and those offered by the GAT, confirming the good relationships between NCT- and GAT-determined IOPs reported by others [33]. These results indicate that the three NCTs provide statistically significant predictions of G-IOP measurements.

Many variables affect IOP measurements, including astigmatism, corneal thickness, corneal biomechanics/hysteresis, accommodation, respiration, heart rate and rhythm [34], and diurnal variation [26]. Considering these sources of error, some authors advocate that $\pm 3.0 \mathrm{mmHg}$ should be the maximum clinically acceptable error [19]. 
Table 2 Pairwise comparative analyses between each NCT and GAT in subgroups divided by G-IOP

\begin{tabular}{lllll}
\hline Subgroups $^{\mathrm{a}}$ & $\mathrm{n}$ & $\mathrm{C}-\mathrm{IOP}-\mathrm{G}-\mathrm{IOP}$ & $\mathrm{N}-\mathrm{IOP}-\mathrm{G}-\mathrm{IOP}$ & $\mathrm{T}-\mathrm{IOP}-\mathrm{G}-\mathrm{IOP}$ \\
\hline Low-teen & 14 & $\mathbf{1 . 7 1} \pm \mathbf{2 . 2 3}$ & $-1.14 \pm 2.57$ & $1.14 \pm 1.96$ \\
& & $(\boldsymbol{p}=\mathbf{0 . 0 1 3})$ & $(p=0.120)$ & $(p=0.048)$ \\
Mid-teen & 22 & $\mathbf{1 . 7 7} \pm \mathbf{1 . 3 4}$ & $-0.32 \pm 1.67$ & $1.00 \pm 2.07$ \\
& & $(\boldsymbol{p}<\mathbf{0 . 0 0 1 )}$ & $(p=0.382)$ & $(p=0.034)$ \\
High-teen & 16 & $0.56 \pm 1.59$ & $\mathbf{- 1 . 7 5} \pm \mathbf{1 . 6 5}$ & $-0.81 \pm 1.83$ \\
& & $(p=0.178)$ & $(\boldsymbol{p}=\mathbf{0 . 0 0 1 )}$ & $(p=0.097)$ \\
\hline
\end{tabular}

$C$-IOP IOP value measured by Canon NCT, N-IOP Nidek NCT, T-IOP Topcon NCT, G-IOP GAT

Values are presented as the mean \pm standard deviation. P value by paired t-test

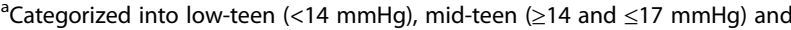
high-teen $(>17 \mathrm{mmHg}$ ) groups

In our study, according to the Bland-Altman plots, the 95\% LoA between C-IOP and G-IOP were close to this clinically acceptable error $( \pm 3.43 \mathrm{mmHg}), 92 \%$ of the differences falling within this range. This means that the $\mathrm{N}$ IOP showed $8 \%$ clinical error in our study subjects, all of whose IOP was within the normal range. In case of $\mathrm{N}$ IOP, the LoA were wider $( \pm 3.92 \mathrm{mmHg})$, and $89 \%$ of the differences were within the clinically acceptable limits. The LoA of T-IOP was the widest $( \pm 4.16 \mathrm{mmHg})$, and $87 \%$ of the differences were within the clinically acceptable limits. These differences of LoA among the three NCTs might be accounted for in the differences in the alignment methods: the Canon TX-20P and Topcon CT-1P make parallel automatically, whereas the Nidek NT-530P aligns manually. Manual alignments might allow the operator to more accurately adjust subjects' cornea in the three, axial, vertical and lateral dimensions. The reason for the narrower LoA of the Canon TX-20P than that of the Nidek NT-530P should be clarified by further evaluation with a larger subject cohort.

While there was a statistically significant difference between C-IOP and G-IOP as well as between N-IOP and G-IOP, none such was detected between T-IOP and GIOP, suggesting that any such differences as might exist are independent of the mean IOP values. Thus, we could argue that the Topcon CT-1P offers relatively closer agreement with the GAT than does the Canon TX-20P or Nidek NT-530P. Furthermore, in the subgroup comparison study by G-IOP, the low- and mid-teen subgroups between C-IOP and G-IOP and the high-teen subgroup between N-IOP and G-IOP showed significant mean differences, demonstrating the tendency of discrepancy between the Canon TX-20P and GAT as well as between the Nidek NT-530P and GAT in these GIOP ranges.

There are some limitations to this study. The fact that we did not test the instruments across the entire useful range is the main one. We also excluded subjects with irregular astigmatism or astigmatism of more than 3.5 diopters, and thus, our findings may hold true only for subjects with similar refraction characteristics. Furthermore, the sample size of this study was relatively small for the subgroup analysis; future studies will need to include larger populations of ocular hypertensive and glaucoma subjects, with or without irregular/severe astigmatism, for whom any bias between instruments could have clinical implications. Additionally, we were unable to randomize the order in which the NCTs were used due to restraints on access to the equipment. Therefore, we were unable to avoid potential systematic errors and are unable to ensure that the lack of randomization did not influence the outcome. We did implement a five-minute interval between each measurement to minimize the 'massage effect'. One other limitation was the fact that we did not carry out CCT correction separately with each tonopachymeter; future studies should also investigate the results of CCT correction and comparison of CCT values from the ultrasound pachymeter with those from each tonopachymeter.

In conclusion, this comparative study shows that the only Canon TX-20P provides reliable and repeatable IOP measurements not influenced by CCT within a relatively normal/restricted range of corneal thickness. However, without CCT correction, the Canon TX-20P, Nidek NT-530P and Topcon CT-1P all offer similar accuracy to the GAT. Despite the relatively small sample size of this study and the absence of high IOPs, the instruments demonstrated good performance in measuring IOP in ocular normotensive non-glaucomatous subjects. These results suggest the instruments will prove useful for rapid IOP testing in clinical practice. Indeed, practitioners should keep in mind the differences in the tendencies of the IOP values as measured by the respective NCTs.

\section{Conclusion}

Without CCT correction, the Canon TX-20P, Nidek NT530P and Topcon CT-1P all offer similar accuracy to the GAT.

\section{Abbreviations \\ C-IOP: Intraocular pressure values obtained with the Canon TX-20P; CCT: Central corneal thickness; D: Diopters; GAT: Goldmann applanation tonometer; G-IOP: Intraocular pressure values obtained with the GAT; ICC: Intraclass correlation coefficient; IOP: Intraocular pressure; IRB: Institutional Review Board; LoA: Limits of agreement; NCT: Non-contact tonometers; N-IOP: Intraocular pressure values obtained with the Nidek NT- 530P; SD: Standard deviation; T-IOP: Intraocular pressure values obtained with the Topcon CT-1P}

\section{Acknowledgments}

A summary of this paper was presented as a narrative at the 115th Spring Meeting of the Korean Ophthalmology Society, Busan, Korea, April 9-10, 2016.

\section{Funding}

This work was supported the National Research Foundation of Korea (NRF) grant funded by the Korean government (MSIP; no. 2014R1A5A2010008). The sponsor had no role in the design or conduct of this research. 


\section{Availability of data and materials}

All data are available upon request.

\section{Authors' contributions}

SPB, CEL and YCK were responsible for the conception and design of this study. SPB and CEL acquired, analyzed and interpreted the data. SPB drafted the manuscript. CEL and YCK revised the manuscript critically for important intellectual content. All authors have read and approved the final manuscript and agree to be accountable for all aspects of the work in ensuring that questions related to the accuracy or integrity of any part of the work are appropriately investigated and resolved. All authors read and approved the final manuscript.

\section{Ethics approval and consent to participate}

This was a retrospective study; therefore, informed written informed consent could not be obtained from the patients. Nevertheless, all patient data were anonymized. Moreover, verbal informed consent was obtained from all patients before examination. This study was approved by the Institutional Review Board (IRB) of Keimyung University Dongsan Medical Center (IRB no. 2015-12-052) and was performed in accordance with the tenets of the Declaration of Helsinki.

\section{Consent for publication}

Not applicable.

\section{Competing interests}

The authors declare that they have no competing interests.

\section{Publisher's Note}

Springer Nature remains neutral with regard to jurisdictional claims in published maps and institutional affiliations.

Received: 22 May 2017 Accepted: 27 October 2017

Published online: 02 November 2017

\section{References}

1. Hansen MK. Clinical comparison of the XPERT non-contact tonometer and the conventional Goldmann applanation tonometer. Acta Ophthalmol Scand. 1995;73(2):176-80

2. Beatty S, Nischal KK, Jones $H$, Eagling EM. Effect of applanation tonometry on mean corneal curvature. J Cataract Refract Surg. 1996;22(7):970-1.

3. Cho P, Lui T. Comparison of the performance of the Nidek NT-2000 noncontact tonometer with the Keeler Pulsair 2000 and the Goldmann applanation tonometer. Optom Vis Sci. 1997;74(1):51-8

4. Jorge J, Diaz-Rey JA, Gonzalez-Meijome JM, Almeida JB, Parafita MA. Clinical performance of the Reichert AT550: a new non-contact tonometer. Ophthalmic Physiol Opt. 2002;22(6):560-4.

5. Garcia Resua C, Giraldez Fernandez MJ, Cervino Exposito A, Gonzalez Perez J, Yebra-Pimentel E. Clinical evaluation of the new TGDc-01 "PRA" palpebral tonometer: comparison with contact and non-contact tonometry. Optom Vis Sci. 2005:82(2):143-50

6. van der Jagt $\mathrm{LH}$, Jansonius NM. Three portable tonometers, the TGDc-01, the ICARE and the Tonopen $\mathrm{XL}$, compared with each other and with Goldmann applanation tonometry. Ophthalmic Physiol Opt. 2005;25(5): 429-35

7. Shields MB. The non-contact tonometer. Its value and limitations. Surv Ophthalmol. 1980;24(4):211-9.

8. Moreno-Montanes J, Gomez-Demmel E, Lajara-Blesa J, Aliseda-Perez de Madrid D. Comparative study of three non-contact tonometers and the Goldmann tonometer. Ophthalmologica. 1994;208(3):115-8.

9. Tonnu PA, Ho T, Sharma K, White E, Bunce C, Garway-Heath D. A comparison of four methods of tonometry: method agreement and interobserver variability. Br J Ophthalmol. 2005;89(7):847-50.

10. Jansson U, Brautaset $R$, Cerviño A, Nilsson M. A comparison of the canon TX-20P'M non-contact tonometer and pachymeter in healthy eyes. Int J Ophthalmic Pract. 2012;3(3):96-102.

11. Tranchina L, Lombardo M, Oddone F, Serrao S, Schiano Lomoriello D, Ducoli $P$. Influence of corneal biomechanical properties on intraocular pressure differences between an air-puff tonometer and the Goldmann applanation tonometer. J Glaucoma. 2013;22(5):416-21.
12. Anton A, Neuburger M, Bohringer D, Jordan JF. Comparative measurement of intraocular pressure by lcare tonometry and Airpuff tonometry in healthy subjects and patients wearing therapeutic soft contact lenses. Graefes Arch Clin Exp Ophthalmol. 2013;251(7):1791-5.

13. Wei W, Fan Z, Wang L, Li Z, Jiao W, Li Y. Correlation analysis between central corneal thickness and intraocular pressure in juveniles in northern China: the Jinan city eye study. PLoS One. 2014;9(8):e104842.

14. Garcia-Resua C, Pena-Verdeal H, Minones M, Giraldez MJ, Yebra-Pimentel E. Reliability of the non-contact tono-pachymeter Tonopachy NT-530P in healthy eyes. Clin Exp Optom. 2013;96(3):286-94.

15. Fujimura F, Kamiya K, Fujiwara K, Shoji N, Shimizu K. Repeatability and reproducibility of measurements using a NT-530P noncontact tono/ pachymeter and correlation of central corneal thickness with intraocular pressure. Biomed Res Int. 2013;2013:370592.

16. Ogbuehi KC. Assessment of the accuracy and reliability of the Topcon CT80 non-contact tonometer. Clin Exp Optom. 2006;89(5):310-4.

17. Farhood QK. Comparative evaluation of intraocular pressure with an air-puff tonometer versus a Goldmann applanation tonometer. Clin Ophthalmol. 2013;7:23-7.

18. Tamcelik N, Atalay E, Cicik E, Ozkok A. Comparability of Icare pro rebound Tonometer with Goldmann Applanation and noncontact Tonometer in a wide range of intraocular pressure and central corneal thickness. Ophthalmic Res. 2015;54(1):18-25

19. Garcia-Resua C, Giraldez Fernandez MJ, Yebra-Pimentel E, Garcia-Montero S. Clinical evaluation of the canon TX-10 noncontact tonometer in healthy eyes. Eur J Ophthalmol. 2010;20(3):523-30.

20. Pelit A, Altan-Yaycioglu R, Pelit A, Akova YA. Effect of corneal thickness on intraocular pressure measurements with the Pascal dynamic contour, canon TX-10 non-contact and Goldmann applanation tonometers in healthy subjects. Clin Exp Optom. 2009;92(1):14-8.

21. Yilmaz I, Altan C, Aygit ED, Alagoz C, Baz O, Ahmet S, Urvasizoglu S, Yasa D, Demirok A. Comparison of three methods of tonometry in normal subjects: Goldmann applanation tonometer, non-contact airpuff tonometer, and Tono-pen XL. Clin Ophthalmol. 2014;8:1069-74.

22. Esen F, Kostek M, Emekli AS, Eraslan M. Double-organ bias in published randomized controlled trials of glaucoma. J Glaucoma. 2016;25(6):520-2.

23. Almubrad TM, Ogbuehi KC. Clinical investigation of the effect of topical anesthesia on intraocular pressure. Clin Ophthalmol. 2007;1(3):305-9.

24. AlMubrad TM, Ogbuehi KC. The effect of repeated applanation on subsequent IOP measurements. Clin Exp Optom. 2008;91(6):524-9.

25. Almubrad TM, Ogbuehi KC. On repeated corneal applanation with the Goldmann and two non-contact tonometers. Clin Exp Optom. 2010;93(2): $77-82$.

26. Liu JH, Bouligny RP, Kripke DF, Weinreb RN. Nocturnal elevation of intraocular pressure is detectable in the sitting position. Invest Ophthalmol Vis Sci. 2003:44(10):4439-42.

27. Doughty MJ, Zaman ML. Human corneal thickness and its impact on intraocular pressure measures: a review and meta-analysis approach. Surv Ophthalmol. 2000;44(5):367-408.

28. Hwang YH, Kim HK, Sohn YH, Namil Study Group KGS. Central corneal thickness in a Korean population: the Namil study. Invest Ophthalmol Vis Sci. 2012;53(11):6851-5.

29. Regine F, Scuderi GL, Cesareo M, Ricci F, Cedrone C, Nucci C. Validity and limitations of the Nidek NT-4000 non-contact tonometer: a clinical study. Ophthalmic Physiol Opt. 2006;26(1):33-9.

30. Recep OF, Hasiripi H, Cagil N, Sarikatipoglu H. Relation between corneal thickness and intraocular pressure measurement by noncontact and applanation tonometry. J Cataract Refract Surg. 2001;27(11):1787-91.

31. Stodtmeister R. Applanation tonometry and correction according to corneal thickness. Acta Ophthalmol Scand. 1998;76(3):319-24

32. Babalola OE, Kehinde AV, Iloegbunam AC, Akinbinu T, Moghalu C, Onuoha I. A comparison of the Goldmann applanation and non-contact (Keeler Pulsair EasyEye) tonometers and the effect of central corneal thickness in indigenous African eyes. Ophthalmic Physiol Opt. 2009;29(2):182-8.

33. Lee YG, Kim JH, Kim NR, Kim CY, Lee ES. Comparison between Tonopachy and other tonometric and pachymetric devices. Optom Vis Sci. 2011;88(7): 843-9.

34. Whitacre MM, Stein R. Sources of error with use of Goldmann-type tonometers. Surv Ophthalmol. 1993;38(1):1-30. 